\title{
OPEN Inhibitory effects of aprotinin on influenza $A$ and $B$ viruses in vitro and in vivo
}

\author{
Eun-Jung Song ${ }^{1,2,5}$, Erica Españo ${ }^{1,5}$, Sang-Mu Shim ${ }^{3,5}$, Jeong-Hyun $\mathrm{Nam}^{1,3}$, Jiyeon $\mathrm{Kim}^{1}$, \\ Kiho Lee ${ }^{1}$, Song-Kyu Park ${ }^{1}$, Chong-Kil Lee ${ }^{4 \bowtie}$ \& Jeong-Ki Kim ${ }^{1 凶}$
}

Influenza viruses cause significant morbidity and mortality worldwide. Long-term or frequent use of approved anti-influenza agents has resulted in drug-resistant strains, thereby necessitating the discovery of new drugs. In this study, we found aprotinin, a serine protease inhibitor, as an antiinfluenza candidate through screening of compound libraries. Aprotinin has been previously reported to show inhibitory effects on a few influenza A virus (IAV) subtypes (e.g., seasonal H1N1 and H3N2). However, because there were no reports of its inhibitory effects on the other types of influenza viruses, we investigated the inhibitory effects of aprotinin in vitro on a wide range of influenza viruses, including avian and oseltamivir-resistant influenza virus strains. Our cell-based assay showed that aprotinin had inhibitory effects on seasonal human IAVs (H1N1 and H3N2 subtypes), avian IAVs (H5N2, H6N5, and H9N2 subtypes), an oseltamivir-resistant IAV, and a currently circulating influenza $B$ virus. We have also confirmed its activity in mice infected with a lethal dose of influenza virus, showing a significant increase in survival rate. Our findings suggest that aprotinin has the capacity to inhibit a wide range of influenza virus subtypes and should be considered for development as a therapeutic agent against influenza.

Influenza viruses remain important pathogens that cause respiratory diseases in humans and animals. Human seasonal influenza A and B viruses annually cause severe morbidity worldwide. The Centers for Disease Control (CDC) estimates around 23,000 flu-related deaths in the United States each year ${ }^{1}$. In addition, avian influenza viruses, such as the H5 and H7 subtypes, sporadically cause highly lethal infections in both animals and humans $s^{2-4}$, and animal or human-animal influenza reassortant strains occasionally cause global epidemics or pandemic influenza ${ }^{5}$.

Vaccination is considered the most effective strategy for controlling influenza in humans ${ }^{6}$. However, current influenza vaccines have several limitations, including their limited efficacy due to antigenic mismatches between the vaccine and circulating virus strains ${ }^{7}$. For this reason, antiviral drugs are important for controlling influenza. Representative classes of anti-influenza drugs include adamantane-based matrix protein 2 (M2) ion channel blockers (e.g., amantadine and rimantadine) and neuraminidase (NA) inhibitors (e.g., oseltamivir and zanamivir $)^{8}$. However, the emergence of antiviral drug resistance is a constant concern, owing to the high mutation rates of influenza viruses through antigenic drift and shift ${ }^{9}$. Since the first report of amantadine-resistant influenza A viruses (IAVs) during the 1980 epidemic $^{10}$, the prevalence of these viruses among circulating IAVs (especially, H1N1 and H3N2 subtypes) has increased rapidly to nearly $100 \%$ of the cases ${ }^{11}$. In response, the CDC has stopped recommending the use of adamantane in the United States ${ }^{12}$. Increasing application of NA inhibitors (especially oseltamivir) brings into focus the risk of developing resistance to this class of anti-influenza drugs. Although the prevalence of NA inhibitor-resistant influenza viruses is generally low (oseltamivir $<3.5 \%$ ) or rare (zanamivir $<1 \%)^{13-16}$, the problem of reduced susceptibility and resistance of influenza viruses to NA inhibitors has been recently increasing. Therefore, it is an utmost need to develop better or novel anti-influenza drugs to prepare for emergencies.

\footnotetext{
${ }^{1}$ Department of Pharmacy, College of Pharmacy, Korea University, 2511 Sejong-ro, Sejong 30019, Republic of Korea. '2Laboratory Animal Medicine, College of Veterinary Medicine, Chonnam National University, Gwangju 61186, Republic of Korea. ${ }^{3}$ Division of Acute Viral Diseases, Center for Emerging Virus Research, National Institute of Health, Korea Disease Control and Prevention Agency, Cheongju, Chungbuk 28159, Republic of Korea. "Department of Pharmaceutics, College of Pharmacy, Chungbuk National University, Cheongju, Chungbuk 28644, Republic of Korea. ${ }^{5}$ These authors contributed equally: Eun-Jung Song, Erica Españo and Sang-Mu Shim. ${ }^{\boxplus}$ email: cklee@chungbuk.ac.kr; jkfrancis@korea.ac.kr
} 
In this study, we first aimed to identify anti-influenza viral agents by screening compound libraries. Aprotinin, a serine protease inhibitor used to prevent bleeding in cardiopulmonary bypass surgery, presented as a candidate. Previous reports have suggested that aprotinin has anti-influenza virus activity based on its ability to prevent the cleavage of hemagglutinin (HA), a step required for viral-host fusion ${ }^{17-19}$. However, most reports cover only a narrow range of IAV strains (especially seasonal IAV strains) and strains of influenza B virus (IBV) that may no longer be circulating. Furthermore, little is known about the effects of aprotinin against oseltamivir-resistant IAV strains.

Therefore, in this study, we investigated the effects of aprotinin on various subtypes of IAV, including (i) human seasonal IAVs, (ii) avian influenza viruses with zoonotic potential (H5N2, H9N2, and H6N2), (iii) oseltamivir-resistant IAV, and (iv) on a currently circulating strain of IBV in vitro. We also used a mouse model to verify the anti-influenza activity of aprotinin. Our findings contribute further evidence to the potential of aprotinin as a broad-spectrum anti-influenza agent.

\section{Results}

In vitro dose-dependent inhibitory effects of aprotinin on influenza A and B viruses. To develop better or novel antiviral drugs against influenza virus infection, we screened compound libraries through a Madin-Darby canine kidney (MDCK) cell-based screening system ${ }^{20}$ using A/PR/8/34 (H1N1) virus, the standard reference strain of human IAV. We tested a total of 1280 compounds and found 13 anti-influenza candidates. Aprotinin, a serine protease inhibitor, was selected as a final candidate because both aprotinin samples from this library and from that of another company showed inhibitory effects on the virus (data not shown). To validate the results of screening, we treated MDCK cells infected with different influenza virus strains with varying concentrations (10-200 nM) of aprotinin (Fig. 1). Viral inhibition assay in MDCK cells showed that aprotinin inhibits A/PR/8/34 (H1N1) in a dose-dependent manner (Fig. 1a). Aprotinin could also inhibit A/CA/04/09 (H1N1), A/PH/2/82 (H3N2), A/AB/Kor/CN05/09 (H6N5), A/Ck/Kor/01310/01 (H9N2), A/Bris/10/07 (H3N2), and $\mathrm{B} /$ Seoul/32/11 in a dose-dependent manner (Fig. $1 \mathrm{~b}-\mathrm{g}$ ). The calculated $\mathrm{EC}_{50}$ values for aprotinin against the different influenza strains are shown in Table 1 . Based on the results of the viral inhibition assay, we applied the lowest effective concentration against all the tested influenza strains $(60 \mathrm{nM})$ for subsequent experiments.

We next compared the antiviral activity of aprotinin against A/PR/8/34 (H1N1) virus with that of oseltamivir $(100 \mu \mathrm{M})$. Aprotinin showed corresponding or superior antiviral activity to oseltamivir against PR/8 virus infection (Fig. 2a). Colorimetric cytotoxicity assay revealed that there was no cytotoxicity in the range of aprotinin concentrations tested in this study ( $\leq 200 \mathrm{nM})$ (Fig. 2b).

In vitro inhibitory effects of aprotinin on multiple replication cycles of various influenza A virus subtypes. Previous studies have minimal information on the spectrum of the anti-influenza viral activity of aprotinin. Therefore, we evaluated the effects of aprotinin treatment on the production of infectious particles of various IAV subtypes, including human and avian viruses, through time-based studies by determining the growth kinetics of the following viruses in MDCK cells: A/PR/8/34 (H1N1), A/CA/04/09 (H1N1), A/PH/2/82 (H3N2), A/AB/Kor/CN2/09 (H5N2), A/AB/Kor/CN5/09/H6N5 (H6N5), and A/Ck/Kor/01310/09 (H9N2) viruses. Culture supernatants were collected at different time points, and virus titers were determined by calculating the median $\mathrm{TCID}_{50}$ based on the hemagglutination assay. We also compared the inhibitory effects of aprotinin against those of oseltamivir $(100 \mu \mathrm{M})$.

Aprotinin was able to significantly reduce the production of the tested human IAVs after more than $16 \mathrm{~h}$ post-infection (Fig. 3a-c), supporting the results of previous studies ${ }^{18,19}$. Furthermore, the inhibitory effects of aprotinin on A/CA/04/09 (H1N1) and A/PH/2/82 (H3N2) viruses were more superior than those of oseltamivir towards the end of the incubation period. Aprotinin was also able to inhibit the production of avian influenza viruses in MDCK cells (Fig. 3d-f). Aprotinin displayed weaker inhibitory effects on avian IAVs than oseltamivir at early time points post-infection but presented effects similar to those of oseltamivir at $64 \mathrm{~h}$ post-infection. These results indicate that aprotinin had inhibitory effects on infections of both human and avian IAVs.

In vitro inhibitory effects of aprotinin on multiple cycles of replication of oseltamivir-resistant influenza $\mathrm{A}$ and $\mathrm{B}$ viruses. We next examined the inhibitory effects of aprotinin against an oseltamivirresistant IAV (A/Bris/10/07; H3N2). As shown in Fig. 4a, A/Bris/10/07 (H3N2) virus was less susceptible to oseltamivir. Our genetic analysis revealed that the H28T mutation in HA conferred the reduced susceptibility of the $\mathrm{H} 3 \mathrm{~N} 2$ virus to oseltamivir (data not shown). However, aprotinin could effectively reduce the production of the $\mathrm{H} 3 \mathrm{~N} 2$ virus (Fig. 4a).

IBVs are generally less susceptible to oseltamivir than IAVs ${ }^{21,22}$. Therefore, we investigated the inhibitory effects of aprotinin on influenza B virus (B/Seoul/32/2011) infection. As shown in Fig. 4b, the IBV was around 50 - to 100 -fold less susceptible to oseltamivir. However, aprotinin was more effective than oseltamivir at reducing IBV production.

Taken together, these results suggest that aprotinin can significantly reduce the production of oseltamivirresistant IAV and IBV.

Inhibitory effects of aprotinin treatment in mice infected with a lethal dose of A/PR/8/34 (H1N1) virus. To test whether aprotinin has antiviral activity in vivo, we tested its effects against lethal A/ $\mathrm{PR} / 8 / 34$ (H1N1) virus infection in C57BL/6 mice. We initially treated mice with once-daily intravenous injections of aprotinin at $2 \mathrm{mg} / \mathrm{kg}$ mouse body weight per day based on a previous study ${ }^{23}$. However, while it did not have toxic effects in vivo, it also did not display antiviral effects in influenza-infected mice (data not shown). 

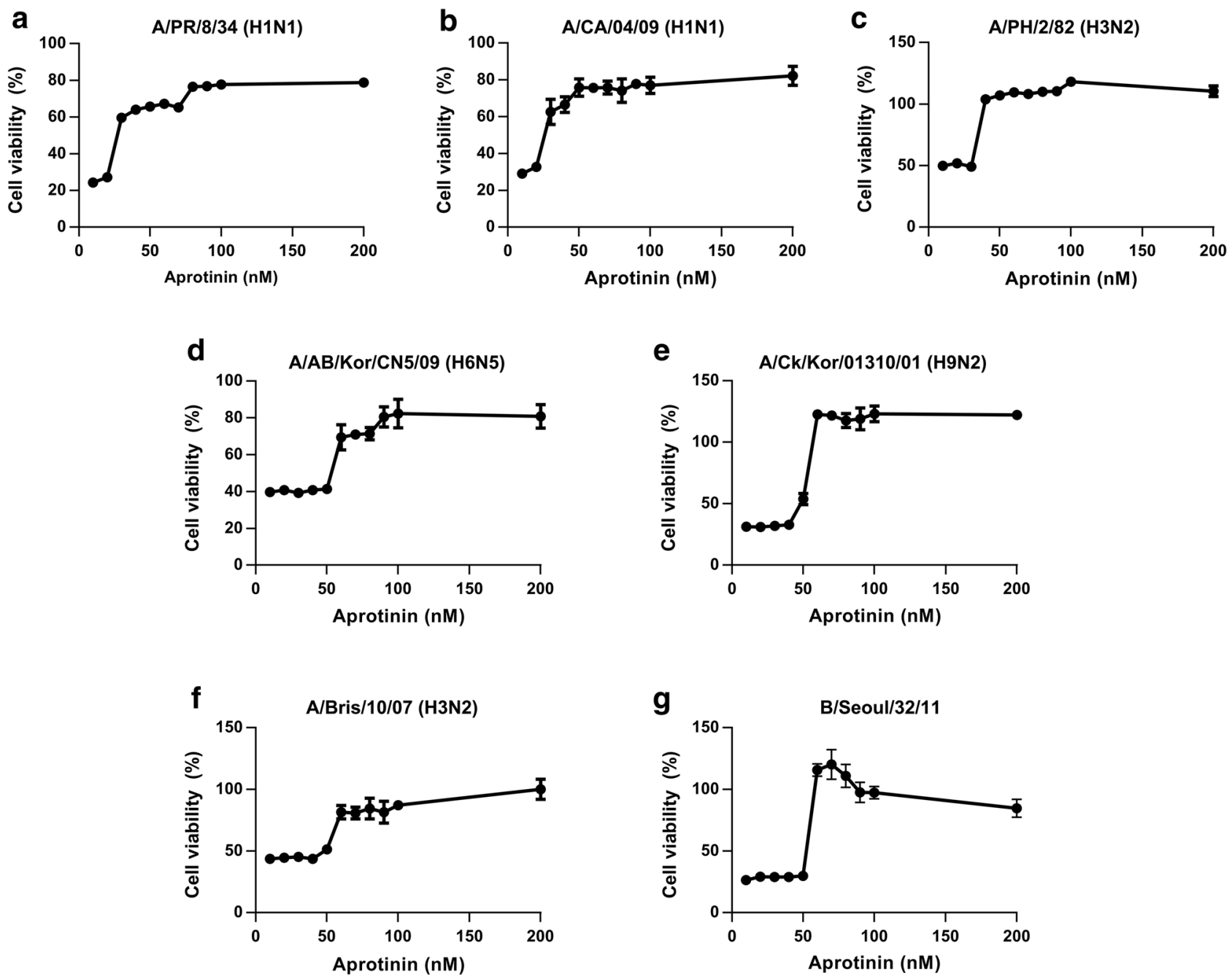

Figure 1. Dose-dependent effects of aprotinin against influenza viruses in vitro. Madin-Darby canine kidney (MDCK) cells were infected with (a) A/PR/8/34 (H1N1), (b) A/CA/04/09 (H1N1), (c) A/PH/2/82 (H3N2), (d) $\mathrm{A} / \mathrm{AB} /$ Kor/CN5/09 (H6N5), (e) A/Ck/Kor/01310/01 (H9N2), (f) A/Bris/10/07 (H3N2), or (g) B/Seoul/32/11 (Yamagata-like lineage) and were treated with varying concentrations of aprotinin $(10-200 \mathrm{nM} ; \mathrm{n}=3$ per dose) for $72 \mathrm{~h}$. Viruses were inoculated at a dose of 100 (H6N5 and B/Seoul) or 1000 (all except H6N5 and B/Seoul) $\mathrm{TCID}_{50}$ per well. Cell viability was measured using the EZ-Cytox reagent, and cell viability was calculated relative to the uninfected MDCK cell viability (cell-only control). TCID 5 : median tissue culture infectious dose.

\begin{tabular}{|l|l|l|}
\hline Type & Virus & EC $_{50}$ \\
\hline A & A/PR/8/34 (H1N1) & $14 \mathrm{nM}$ \\
\hline A & A/CA/04/09 (H1N1, 2009 pandemic) & $11 \mathrm{nM}$ \\
\hline A & A/PH/2/82 (H3N2) & $21 \mathrm{nM}$ \\
\hline A & A/AB/Kor/CN5/09 (H6N5) & $87 \mathrm{nM}$ \\
\hline A & A/Ck/Kor/01310/01 (H9N2) & $57 \mathrm{nM}$ \\
\hline A & A/Bris/10/07 (H3N2, oseltamivir-resistant) & $110 \mathrm{nM}$ \\
\hline B & B/Seoul/32/11 (Yamagata-like) & $39 \mathrm{nM}$ \\
\hline
\end{tabular}

Table 1. Half-maximal effective concentrations $\left(\mathrm{EC}_{50}\right)$ of aprotinin against various influenza virus strains in Madin-Darby canine kidney (MDCK) cells. 

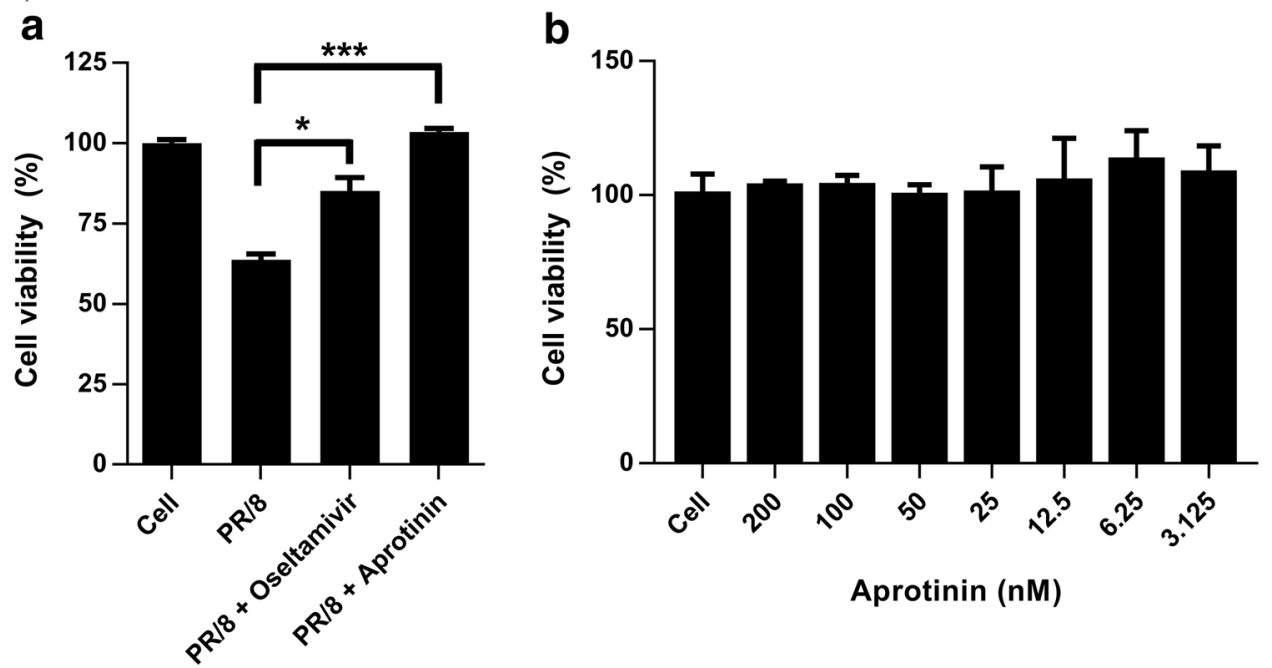

Aprotinin (nM)

Figure 2. Antiviral effects of aprotinin compared with oseltamivir and cytotoxicity assay in Madin-Darby canine kidney (MDCK) cells. (a) MDCK cells were infected with A/PR/8/34 (1000 TCID $5 / \mathrm{ml})$ and treated with $60 \mathrm{nM}$ aprotinin or $100 \mu \mathrm{M}$ oseltamivir. Untreated and uninfected MDCK cells (cell) and untreated infected cells (PR/8) were used as controls. (b) To determine the cytotoxicity of aprotinin, cell viability was measured by treating the MDCK cells with the compound for $72 \mathrm{~h}$, and cell viability was compared with untreated control cells (cell). Cell viability was measured using EZ-Cytox. The experiments were performed in triplicate. ${ }^{\star} P<0.05$, ${ }^{* *} P<0.01$, and ${ }^{* * *} P<0.001$. TCID ${ }_{50}$ : median tissue culture infectious dose.

The initial half-life of aprotinin may be too short for conferring antiviral effects in vivo. As such, we decided to administer aprotinin twice a day.

$\mathrm{C} 57 \mathrm{BL} / 6$ mice were intranasally inoculated with $3 \mathrm{LD}_{50}$ of $\mathrm{A} / \mathrm{PR} / 8 / 34(\mathrm{H} 1 \mathrm{~N} 1)$ virus. For 5 days after infection, the mice received twice-daily intravenous injections of aprotinin $(2 \mathrm{mg} / \mathrm{kg}$ mouse body weight, twice daily); oral administrations of oseltamivir $\left(10 \mathrm{mg} / \mathrm{kg} /\right.$ day) based on a previously reported effective dose ${ }^{24}$; or phosphatebuffered saline (PBS) as control. Uninfected mice were similarly treated with aprotinin as the drug-only control group. Body weight changes and survival were monitored daily for 14 days after infection (Fig. 5). Administration of aprotinin alone did not result in body weight loss in mice. The PBS-treated mice had $0 \%$ survival at 8 days post-infection. Meanwhile, the groups of mice treated with either aprotinin and oseltamivir showed $75 \%$ and $100 \%$ survival, respectively.

\section{Discussion}

Given the limitations of influenza vaccines and the recent rise in the number of oseltamivir-resistant strains, there remains a need to discover and develop new anti-influenza agents. In our cell culture-based screening of compound libraries, aprotinin was identified as a strong anti-influenza candidate. Aprotinin, also known as bovine pancreatic trypsin inhibitor, is a naturally occurring non-specific inhibitor of serine proteases, including trypsin, chymotrypsin, plasmin, and kallikrein ${ }^{25}$. It is primarily indicated for preventing blood loss in cardiac bypass surgeries. It was considered to be well-tolerated in animal models and in clinical trials ${ }^{26}$. Aprotinin has been previously reported as an anti-influenza agent in vitro ${ }^{27}$, in embryonated chicken eggs ${ }^{17,28}$, and in mice $^{29}$. It is currently licensed in Russia for clinical use in aerosolized form (Aerus), primarily against seasonal H1N1 and $\mathrm{H} 3 \mathrm{~N} 2$ influenza, but it has also been tested against $\mathrm{H} 2 \mathrm{~N} 2$, pandemic $\mathrm{H} 1 \mathrm{~N} 1$, and avian-like H7N9 influenza viruses $^{18,19,28}$.

Influenza viruses require proteolytic cleavage and structural rearrangement of HA for successful fusion with host endosomes. HA is initially translated as a precursor, HA0, with HA1 and HA2 domains linked by a short peptide sequence. Trypsin-like proteases facilitate cleavage of the precursor by targeting arginine in the linker peptide in the HAO of most influenza virus strains. Extracellular cleavage of the HA0 of mammalian and low-pathogenicity influenza viruses is facilitated by soluble proteases such as tryptase Clara, mini-plasmin, and ectopic anionic trypsin $\mathrm{I}^{30}$. Cell-associated cleavage of HA0 in the human airway can also be facilitated by transmembrane protease serine 2 (TMPRSS2) in secretory vesicles during viral egress and by the membrane-bound human airway-trypsin like protease (HAT) prior to attachment to target cells ${ }^{30,31}$. Aprotinin inhibits the activity of trypsin-like proteases by blocking the active site, thereby inhibiting proteolytic cleavage of $\mathrm{HA} 0^{28}$. Aprotinin also displayed inhibitory effects on paramyxoviruses ${ }^{32}$ and SARS-CoV- $2^{33}$, likely due to suppressed cleavage of the fusion protein precursor and the spike protein, respectively.

Because previous studies have shown that aprotinin inhibited a limited number of subtypes of IAV (mainly H1N1 and H3N2), we decided to examine its antiviral activity against a broader range of influenza viruses in this study. The tested strains include avian strains of IAV, an oseltamivir-resistant strain of IAV, and a currently circulating strain of IBV. 
a

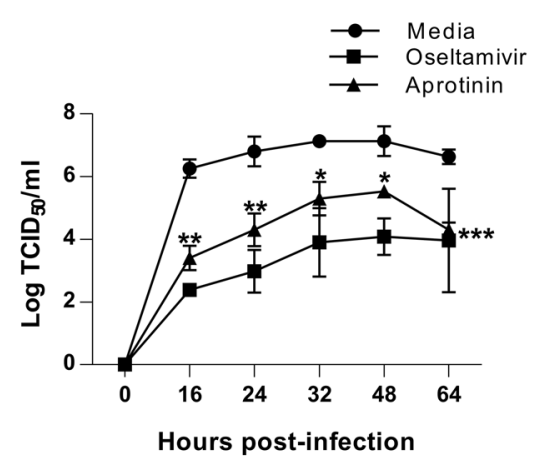

d

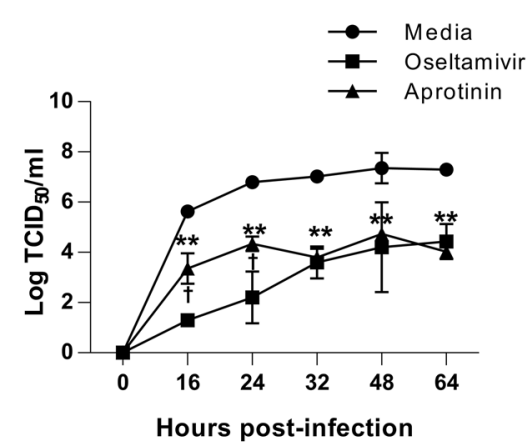

b

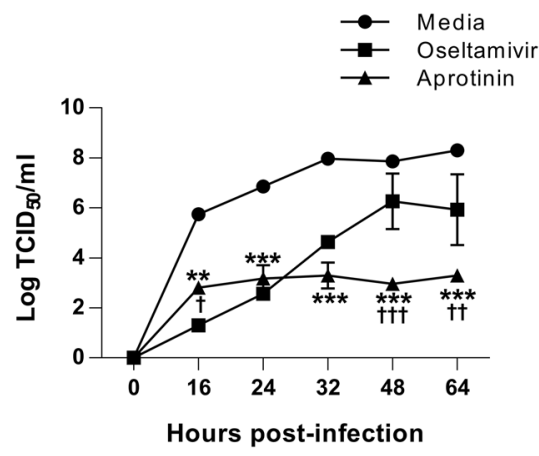

e

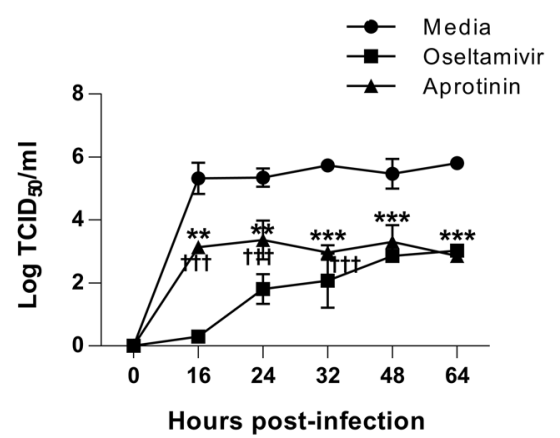

C

A/PH/2/82 (H3N2)
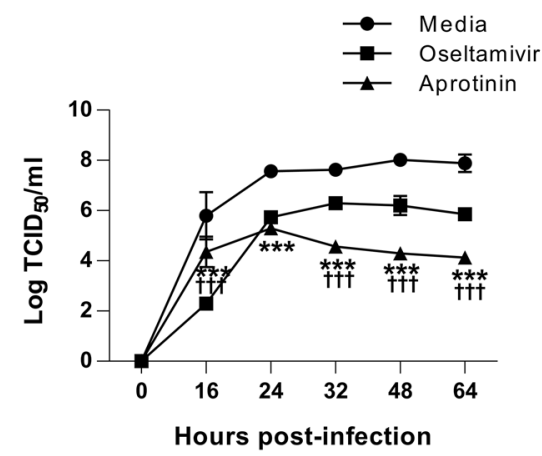

$\mathbf{f}$

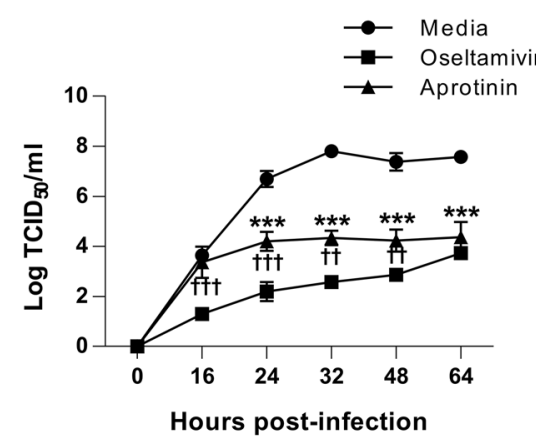

Figure 3. Aprotinin inhibited the replication of various strains of human influenza A virus in Madin-Darby canine kidney (MDCK cells. The replication kinetics of (a) A/PR/8/34 (H1N1), (b) A/CA/04/09 (H1N1), (c) A/ $\mathrm{PH} / 2 / 82$ (H3N2), (d) A/AB/Kor/CN2/09 (H5N2), (e) A/AB/Kor/CN5/09 (H6N5), and (f) A/Ck/Kor/01310/01 (H9N2) virus were investigated in MDCK cells after treatment with aprotinin and oseltamivir. MDCK cells were infected with influenza virus at an MOI of 0.001 (H6N5) or 0.01 (all viruses except H6N5) for $1 \mathrm{~h}$ and then treated with aprotinin $(60 \mathrm{nM})$ or oseltamivir $(100 \mu \mathrm{M})$. Supernatants were collected pre-infection $(0)$ and at $16,24,32,48$, and $64 \mathrm{~h}$ post-infection, and viral titers in the supernatants were determined by the $\mathrm{TCID}_{50}$ assay. ${ }^{\star} P<0.05,{ }^{*} P<0.01$, and ${ }^{* *} P<0.0001$, statistically significant difference between the virus-only (media) group and the aprotinin treatment group. ${ }^{\dagger} P<0.05,{ }^{\dagger \dagger} P<0.01$, and ${ }^{\dagger \dagger} P<0.0001$, statistically significant difference between the aprotinin and oseltamivir treatment group. MOI: multiplicity of infection; $\mathrm{TCID}_{50}$ : half-maximal tissue culture infectious dose.

Similar to previous reports, we found that aprotinin was able to inhibit the production of seasonal H1N1 and H3N2 IAVs in MDCK cells. The effects of aprotinin were either comparable or superior to the effects of oseltamivir. We also found that aprotinin could inhibit avian IAVs belonging to the H9N2, H5N2, and H6N5 subtypes in vitro at levels similar to the effects of oseltamivir. H9N2 currently circulates in poultry and is generally avirulent or low-pathogenic. However, occasional outbreaks in poultry farms have occurred, and sporadic human infection cases have also been reported ${ }^{34}$. Meanwhile, both the H5N2 and H6N5 viruses in this study were isolated from wildfowl in South Korea. The H6N5 isolate was found to cause considerable morbidity and mortality in mice without bearing any known pathogenicity marker ${ }^{35}$, and the H5N2 isolate adapted to and caused lethality in mice after only a single lung-to-lung passage ${ }^{36}$. These previous studies suggest that some of the currently circulating avian influenza virus strains have the capacity to easily cross the avian-mammalian transmission barrier and may emerge as zoonotic agents in the future. The ability of aprotinin to inhibit these avian influenza viruses suggests that aprotinin may potentially be used in human outbreaks of avian influenza viruses.

We have also shown that aprotinin is able to inhibit an oseltamivir-resistant influenza A strain (A/Bris/10/07; $\mathrm{H} 3 \mathrm{~N} 2$ ). Additionally, similar to earlier reports of aprotinin's activity against the $\mathrm{B} / \mathrm{Lee} / 40$ and $\mathrm{B} / \mathrm{HK} / 73$ viruses ${ }^{17}$, aprotinin shows antiviral activity against a currently circulating strain of IBV (Yamagata-like lineage, B/ Seoul/32/2011). IBVs are generally less susceptible to oseltamivir, especially in children ${ }^{21,37}$. Because aprotinin targets a host factor required for infection, influenza viruses are less likely to develop aprotinin resistance, especially because trypsin-like proteases, the targets of aprotinin, are required for influenza virus proliferation. Therefore, the use of aprotinin may be more beneficial in the long run than the use of drugs targeted against viral components.

In our study, at least twice-daily intravenous administrations were needed for aprotinin to be protective against influenza virus infection in a mouse model. Aprotinin has a relatively short plasma half-life $(0.7-2 \mathrm{~h})$, and $90 \%$ of the administered dose is absorbed by the kidney in a few hour ${ }^{38}$, which requires high-dose intravenous administrations of aprotinin in surgeries ${ }^{26}$. This probably explains why once-daily intravenous administrations were not sufficient to exert inhibitory effects against the influenza virus. As such, high plasma concentrations of 
a

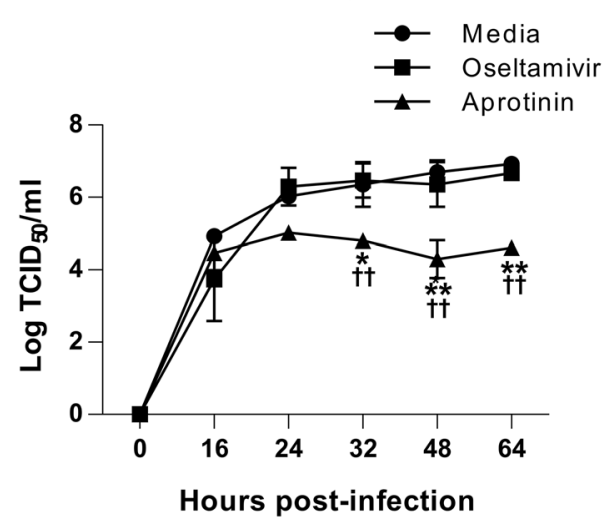

b
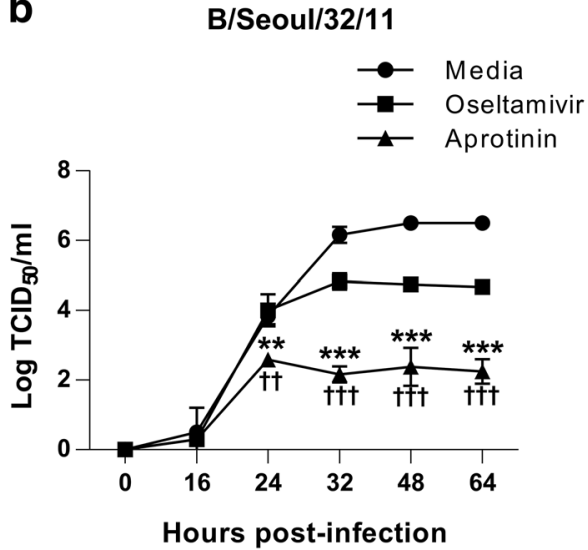

Figure 4. Aprotinin inhibited the replication of oseltamivir-resistant influenza viruses in Madin-Darby canine kidney (MDCK) cells. The replication kinetics of (a) A/Bris/10/07 (H3N2) and (b) B/Seoul/32/11 were investigated in MDCK cells after treatment with aprotinin and oseltamivir. MDCK cells were infected with influenza virus at an $\mathrm{MOI}$ of $0.01(\mathrm{H} 3 \mathrm{~N} 2)$ or $0.001(\mathrm{~B} / \mathrm{Seoul} / 32 / 11)$ for $1 \mathrm{~h}$ and then treated with aprotinin $(60 \mathrm{nM})$ or oseltamivir $(100 \mu \mathrm{M})$. Supernatants were collected pre-infection (0) and at 16, 24, 32, 48, and $64 \mathrm{~h}$ post-infection, and viral titers in the supernatants were determined by the $\operatorname{TCID}_{50}$ assay. ${ }^{\star} P<0.05,{ }^{\star}{ }^{\star} P<0.01$, and ${ }^{* * *} P<0.0001$, statistically significant difference between the virus-only (media) group and the aprotinin treatment group. ${ }^{\dagger} P<0.05,{ }^{\dagger \dagger} P<0.01$, and ${ }^{\dagger+\dagger} P<0.0001$, statistically significant difference between the aprotinin and oseltamivir treatment groups. MOI: multiplicity of infection; $\mathrm{TCID}_{50}$ : half-maximal tissue culture infectious dose.
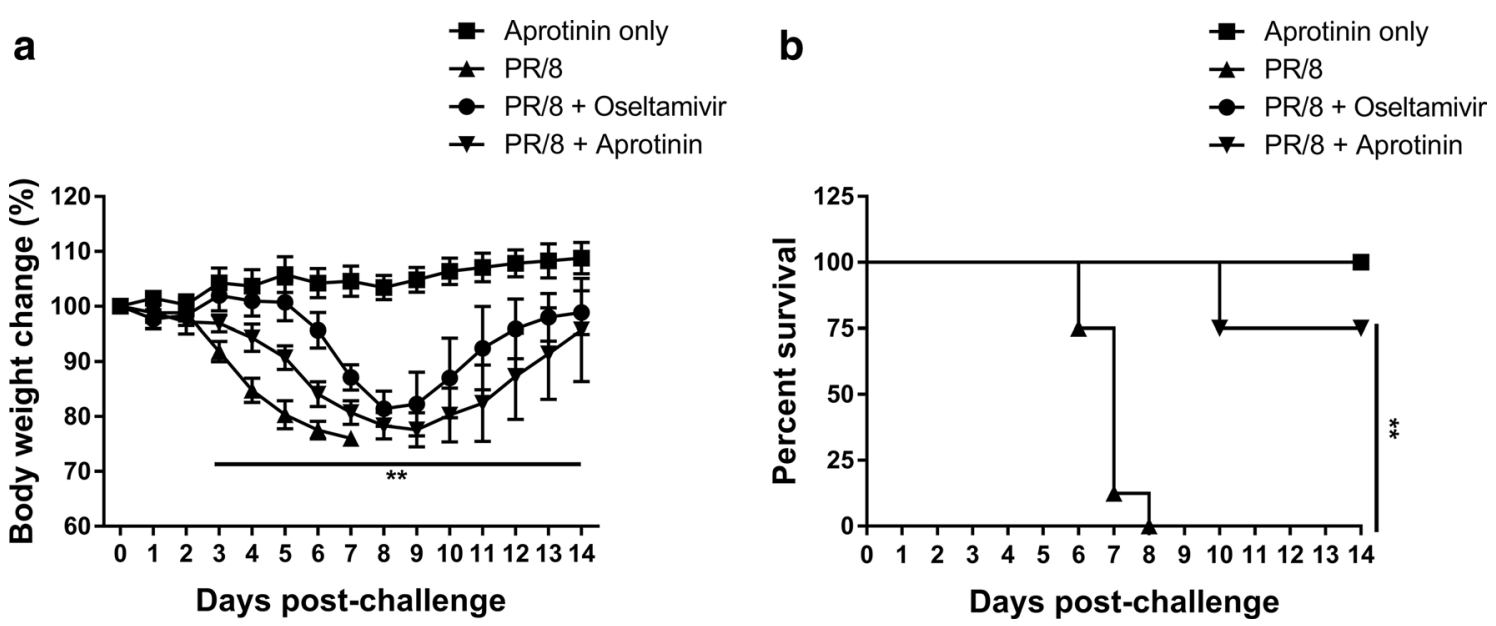

Figure 5. Antiviral effects of aprotinin against influenza A virus in C57BL/6 mice. Groups of mice ( $\mathrm{n}=8$ per group) were intranasally infected with $\mathrm{A} / \mathrm{PR} / 8 / 34(\mathrm{H} 1 \mathrm{~N} 1)$ virus at three times the $50 \%$ mouse lethal dose (3 $\mathrm{LD}_{50}$ ). Oseltamivir was orally administered (oral gavage) at $10 \mathrm{mg} / \mathrm{kg} / \mathrm{day}$, and aprotinin was intravenously administered at $2 \mathrm{mg} / \mathrm{kg}$, twice daily, for 5 days. Mouse (a) body weight changes and (b) survival were monitored daily for 2 weeks. ${ }^{* *} P<0.01$, significant difference between the negative control group (virus-infected only, $\mathrm{PR} / 8)$ and the aprotinin treatment group (PR/8 + aprotinin).

aprotinin may also be required to inhibit influenza viruses. However, as in the case of the licensed aerosolized aprotinin in Russia, multiple doses of intranasally administered aprotinin may be more beneficial for application against influenza virus infection in humans ${ }^{18,19,29,39}$. This way, aprotinin does not have to circulate systemically and will be targeted in the upper respiratory tract, where most influenza virus subtypes replicate in humans. However, in this study, we did not test intranasal administration of aprotinin. Future studies will have to be performed to determine the optimal dosage and route of administration for human application. Moreover, we did not measure viral titers from the mouse respiratory tract and lungs. Testing the effects of aprotinin on titers of different influenza virus subtypes in the mouse respiratory tract should be considered for further studies. Additionally, whether aprotinin will be effective against highly pathogenic avian influenza viruses (HPAIVs) will have to be evaluated. HPAIVs have multibasic cleavage sites that are more accessible to a wide range of proteases ${ }^{40}$. If aprotinin has the ability to inhibit HPAIVs, then it will be a viable pandemic influenza therapeutic candidate that runs a lower risk of causing drug resistance than currently used antivirals like oseltamivir. 
Several studies have associated the use of aprotinin in cardiac bypass surgery with risks of renal failure and mortality ${ }^{41-43}$. Furthermore, re-exposure to aprotinin within six months has been associated with allergic reactions, including anaphylaxis, with a peak at 4-30 days from last exposure ${ }^{44}$. Consequently, aprotinin was pulled out of the market in 2007. In surgery, aprotinin is typically intravenously administered at an initial dose of $1.0-2.0 \times 10^{6}$ kallikrein inhibitor units (KIU), followed by continuous infusion with $0.25-0.5 \times 10^{6} \mathrm{KIU} / \mathrm{hour}^{45}$. Zhirnov et al. reported that $1.0-3.0 \times 10^{3} \mathrm{KIU}$ per day of aerosolized aprotinin was sufficient against influenza virus infection ${ }^{19}$. Given the nearly 1000 -fold difference in dose and the different routes of administration between the application of aprotinin in cardiac bypass surgery and in influenza treatment, subsequent adverse effects may also differ. Notably, Zhirnov et al. reported that aerosolized aprotinin did not cause allergic reactions in an early clinical trial ${ }^{19}$. Furthermore, several groups have challenged the existing evidence on the adverse effects of aprotinin and have shown that aprotinin does not independently increase the risk of renal failure and mortality $y^{4-48}$. Indeed, after reviewing evidence on the benefits and risks of aprotinin, Canada and Europe have re-licensed aprotinin for application in cardiac bypass surgery ${ }^{45,48}$. Thus, with the potentially broad applicability of aprotinin against influenza viruses and the potential differences in dosage requirements, we believe that further evaluation of aprotinin as a treatment for influenza is merited despite the reported adverse effects for the original indication of aprotinin.

Taken together, in this study, we were able to demonstrate that aprotinin inhibits the in vitro production of (1) avian IAVs with zoonotic potential, (2) oseltamivir-resistant IAV, and (3) currently circulating IBV, which is inherently less susceptible to oseltamivir. Thus, we propose that aprotinin is an excellent candidate for the treatment of most IAVs and IBVs in humans. However, whether aprotinin is similarly effective against HPAIVs will have to be evaluated. The most appropriate route of administration and the optimal dosage for the clinical use of aprotinin against influenza will also have to be determined.

\section{Materials and methods}

Cells, viruses, and aprotinin. Madin-Darby canine kidney (MDCK) cells were cultured in Growth Medium: $1 \times$ Minimum Essential Medium (Invitrogen, Carlsbad, CA, USA) supplemented with 10\% fetal bovine serum (FBS; Gibco, Thermo Fisher Scientific Inc., Waltham, MA, USA), 3\% L-glutamine (Gibco), 0.75\% sodium bicarbonate (Gibco), 1\% MEM vitamin solution (Sigma-Aldrich, St. Louis, MO, USA), $50 \mu \mathrm{g} / \mathrm{ml}$ gentamicin (Gibco), and $1 \%$ antibiotic-antimycotic solution (Gibco).

Seven IAVs and one IBV were used in this study: A/Puerto Rico/8/1934 (A/PR/8/34; H1N1); A/California/04/2009 (A/CA/04/09; H1N1); A/Philippines/2/1982 (A/PH/2/82; H3N2); A/Brisbane/10/2007 (A/ Bris/10/07; H3N2); A/Aquatic Bird/Korea/CN2/2009 (A/AB/Kor/CN2/09; H5N2); A/Aquatic Bird/Korea/ CN5/2009 (A/AB/Kor/CN5/09; H6N5); A/Chicken/Korea/01310/2001 (A/Ck/Kor/01310/01; H9N2); and B/ Seoul/32/2011 (B/Seoul/32/11). The IAVs were grown in 10-day-old embryonated chicken eggs for $48 \mathrm{~h}$ at $35^{\circ} \mathrm{C}$. The allantoic fluid was harvested, and aliquots were stored at $-70^{\circ} \mathrm{C}$ until use. The IBV was propagated in MDCK cells in Infection Medium: $1 \times$ MEM supplemented with $0.3 \%$ bovine serum albumin (Sigma-Aldrich) (instead of FBS) and $1.0 \mu \mathrm{g} / \mathrm{ml}$ tosylsulfonyl phenylalanyl chloromethyl ketone (TPCK)-trypsin (Worthington Biochemical Corporation, Lakewood, NJ, USA) incubated at $37^{\circ} \mathrm{C}, 5 \% \mathrm{CO}_{2}$. After incubation for $72 \mathrm{~h}$, the supernatant was harvested, and aliquots were stored at $-70^{\circ} \mathrm{C}$ until use.

Aprotinin (Sigma-Aldrich) was dissolved in distilled water to make stock solutions, and aliquots were stored at $-20^{\circ} \mathrm{C}$ until use. Oseltamivir phosphate (Tamiflu; Roche, Basel, Switzerland) was reconstituted in PBS and used on the same day. Working solutions of both aprotinin and oseltamivir were prepared by diluting the stock solutions with Infection Medium on the day of use for in vitro assays and with PBS for the in vivo study.

Cell viability and cytotoxicity assays. For the cell viability assay, MDCK cells were cultured on a 96-well plate $\left(2 \times 10^{4}\right.$ cells/well $)$ in Growth Medium. After incubation for $16 \mathrm{~h}$ at $37^{\circ} \mathrm{C}$, the cells were infected with each influenza virus at 100 or 1000 half-maximal tissue culture infectious dose $\left(\mathrm{TCID}_{50}\right) /$ well and were washed with phosphate-buffered saline at $1 \mathrm{~h}$ post-infection. Various concentrations (10 to $200 \mathrm{nM}, \mathrm{n}=3$ per dose) of aprotinin diluted with Infection Medium (similar to the IBV infection medium, with TPCK-trypsin) were added into each well to a final volume of $100 \mu \mathrm{l} /$ well. After incubation for $72 \mathrm{~h}$ at $37^{\circ} \mathrm{C}$, cell viability was determined using the EZ-Cytox kit (Daeillab Service Co., South Korea) according to the manufacturer's instructions. Cell viability was indicated by percentage values compared to the negative control (cells that were infected but not treated with aprotinin). Cytotoxicity of aprotinin was measured similarly as described above, but without infection. Cytotoxicity was presented as $\%$ cell viability relative to the negative control (wells containing cells only).

Virus growth kinetics. MDCK cells were cultured on a 24 -well plate $\left(1.25 \times 10^{5}\right.$ cells/well $)$ in Growth Medium. After incubation for $16 \mathrm{~h}$ at $37^{\circ} \mathrm{C}$, the cells were infected with each influenza virus at a multiplicity of infection (MOI) of 0.01 or 0.001 and were washed with PBS at $1 \mathrm{~h}$ post-infection. The cells were treated with aprotinin $(60 \mathrm{nM} /$ well) or oseltamivir $(100 \mu \mathrm{M} /$ well; positive control) in a total volume of $0.5 \mathrm{ml}$ Infection Medium/well and incubated at $37^{\circ} \mathrm{C}, 5 \% \mathrm{CO}_{2}$. Culture supernatant was collected pre-infection and at $16,24,32$, 48 , and $64 \mathrm{~h}$ post-infection and stored at $-70^{\circ} \mathrm{C}$ until analysis.

Virus titration was performed using MDCK cells. The cells were cultured on a 96 -well plate $\left(2 \times 10^{4}\right.$ cells/ well) in Growth Medium and were infected with $100 \mu$ of serial tenfold dilutions of the culture supernatant in Infection Medium. After incubation for $72 \mathrm{~h}$ at $37^{\circ} \mathrm{C}$, the culture supernatant was harvested to determine virus titration by the hemagglutination assay using $0.5 \%$ chicken red blood cells. The virus titers were determined by calculating the $\mathrm{TCID}_{50}$ using the Reed-Muench method ${ }^{49}$. The typical limit of detection for the $\mathrm{TCID}_{50}$ assay of influenza viruses on MDCK cells is $10^{0.3} \mathrm{TCID}_{50} / \mathrm{ml}$. 
Mouse experiments. All animal experiments were conducted in biosafety level 2-plus facilities at the Korea Research Institute of Bioscience and Biotechnology (KRIBB; Daejeon, South Korea). The study was approved by the Institutional Animal Care and Use Committee (IACUC) of KRIBB (approval no. KRIBB-AEC-10046). Animal care and experiments were carried out according to the guidelines of KRIBB IACUC. The studies were designed and are reported in line with the Animal Research: Reporting of In Vivo Experiments (ARRIVE) guidelines.

Six- to eight-week-old C57BL/6 mice were purchased from Koatech (Pyeongtaek, South Korea), and eight mice per group were used. Each mouse was anesthetized with $100 \mu \mathrm{l}$ of 1:1:8 Zoletil:Rompun:PBS (intraperitoneal). Then, the mice were inoculated via intranasal instillation with 3 times the $50 \%$ lethal dose $\left(3 \mathrm{LD}_{50}\right)$ of the $\mathrm{PR} / 8$ virus in $\mathrm{PBS}(30 \mu \mathrm{l} /$ mouse). The following day, the mice were treated intravenously with aprotinin in PBS ( $2 \mathrm{mg} / \mathrm{kg}$ mouse body weight, twice daily; $0.1 \mathrm{ml} / \mathrm{mouse})$; orally (oral gavage) with oseltamivir in PBS (10 mg/kg mouse body weight per day; $0.2 \mathrm{ml} /$ mouse), or intravenously with PBS (negative control) twice a day for 5 days. Uninfected mice were likewise intravenously administered aprotinin in PBS as the aprotinin-only control group. The mice were monitored daily for 14 days for weight change and mortality.

Data analysis. Based on the inhibition of virus growth, the half-maximal effective concentration $\left(\mathrm{EC}_{50}\right)$ of aprotinin was calculated using GraphPad Prism (version 5; San Diego, CA, USA). This procedure is commonly known as a logistic regression using the following formula: $\mathrm{Y}=1 / 1+10\left(\left[\log \mathrm{EC}_{50}-\log \mathrm{X}\right] \times \mathrm{Hill}_{\text {llope }}\right)$, where $\mathrm{Y}$ represents response (inhibition of virus growth), and $\mathrm{X}$ represents the concentration of aprotinin. Hillslope is the parameter to describe the steepness of the curve. Data analysis was performed using GraphPad Prism for Windows. Student's $t$-test was performed to determine differences between two groups. For the virus growth kinetics data, significant differences among the groups were evaluated by ANOVA followed by Tukey's multiple comparison test. The Gehan-Breslow-Wilcoxon test was used to analyze differences in mouse survival. $P$ values less than $0.05(P<0.05)$ were considered statistically significant.

Received: 18 February 2021; Accepted: 16 April 2021

Published online: 03 May 2021

\section{References}

1. Young-Xu, Y., van Aalst, R., Russo, E., Lee, J. K. H. \& Chit, A. The annual burden of seasonal influenza in the US Veterans Affairs population. PLoS ONE 12, e0169344. https://doi.org/10.1371/journal.pone.0169344 (2017).

2. Peiris, J. S. M., de Jong, M. D. \& Guan, Y. Avian influenza virus (H5N1): A threat to human health. Clin. Microbiol. Rev. 20, $243-267$. https://doi.org/10.1128/cmr.00037-06 (2007).

3. Lee, D.-H., Bertran, K., Kwon, J.-H. \& Swayne, D. E. Evolution, global spread, and pathogenicity of highly pathogenic avian influenza H5Nx clade 2.3.4.4. J. Vet. Sci. 18, 269-280. https://doi.org/10.4142/jvs.2017.18.S1.269 (2017).

4. Yang, L. et al. Genesis and spread of newly emerged highly pathogenic H7N9 avian viruses in Mainland China. J. Virol. 91, e01277-e11217. https://doi.org/10.1128/jvi.01277-17 (2017).

5. Saunders-Hastings, P. R. \& Krewski, D. Reviewing the history of pandemic influenza: Understanding patterns of emergence and transmission. Pathogens 5, 66. https://doi.org/10.3390/pathogens5040066 (2016).

6. World Health Organization. Influenza (seasonal). https://www.who.int/en/news-room/fact-sheets/detail/influenza-(seasonal) (2018).

7. Soema, P. C., Kompier, R., Amorij, J.-P. \& Kersten, G. F. A. Current and next generation influenza vaccines: Formulation and production strategies. Eur. J. Pharm. Biopharm. 94, 251-263. https://doi.org/10.1016/j.ejpb.2015.05.023 (2015).

8. De Clercq, E. Antiviral agents active against influenza A viruses. Nat. Rev. Drug Discov. 5, 1015-1025. https://doi.org/10.1038/ $\operatorname{nrd} 2175$ (2006).

9. Hussain, M., Galvin, H. D., Haw, T. Y., Nutsford, A. N. \& Husain, M. Drug resistance in influenza A virus: The epidemiology and management. Infect. Drug Resist. 10, 121-134. https://doi.org/10.2147/IDR.S105473 (2017).

10. Heider, H. et al. Occurrence of amantadine- and rimantadine-resistant influenza A virus strains during the 1980 epidemic. Acta Virol. 25, 395-400 (1981).

11. Hayden, F. Developing new antiviral agents for influenza treatment: What does the future hold?. Clin. Infect. Dis. 48, S3-S13. https://doi.org/10.1086/591851 (2009).

12. Centers for Disease Control and Prevention. CDC recommends against the use of amantadine and rimantadine for the treatment or prophylaxis of influenza in the United States during the 2005-06 influenza season. https://stacks.cdc.gov/view/cdc/25151 (2006).

13. Gubareva, L. V. et al. Global update on the susceptibility of human influenza viruses to neuraminidase inhibitors, 2015-2016. Antiviral Res. 146, 12-20. https://doi.org/10.1016/j.antiviral.2017.08.004 (2017).

14. Lina, B. et al. Five years of monitoring for the emergence of oseltamivir resistance in patients with influenza A infections in the Influenza Resistance Information Study. Influenza Other Respir. Viruses 12, 267-278. https://doi.org/10.1111/irv.12534 (2018).

15. Centers for Disease Control and Prevention. Influenza antiviral drug resistance. https://www.cdc.gov/flu/treatment/antiviralresist ance.htm (2020).

16. Public Health England. PHE guidance on the use of antiviral agents for the treatment and prophylaxis of seasonal influenza. https:// assets.publishing.service.gov.uk/government/uploads/system/uploads/attachment_data/file/833572/PHE_guidance_antivirals_ influenza_201920.pdf (2019).

17. Zhirnov, O. P., Golyando, P. B. \& Ovcharenko, A. V. Replication of influenza B virus in chicken embryos is suppressed by exogenous aprotinin. Arch. Virol. 135, 209-216. https://doi.org/10.1007/BF01309780 (1994).

18. Zhirnov, O. P., Bokova, N. O., Isaeva, E. I., Varobieva, I. V. \& Malyshev, N. A. Pathogenetic treatment of influenza patients with aerosolized form of aprotinin, a protease inhibitor. BIOpreparations Prev. Diagnosis Treat. 2015, 59-64 (2015).

19. Zhirnov, O. P., Klenk, H. D. \& Wright, P. F. Aprotinin and similar protease inhibitors as drugs against influenza. Antiviral Res. 92, 27-36. https://doi.org/10.1016/j.antiviral.2011.07.014 (2011).

20. Sidwell, R. W. \& Smee, D. F. In vitro and in vivo assay systems for study of influenza virus inhibitors. Antiviral. Res. 48, 1-16. https://doi.org/10.1016/s0166-3542(00)00125-x (2000).

21. Kawai, N. et al. A comparison of the effectiveness of oseltamivir for the treatment of influenza A and influenza B: A Japanese multicenter study of the 2003-2004 and 2004-2005 influenza seasons. Clin. Infect. Dis. 43, 439-444. https://doi.org/10.1086/505868 (2006).

22. Sato, M. et al. Effectiveness of oseltamivir treatment among children with influenza A or B virus infections during four successive winters in Niigata City, Japan. Tohoku J. Exp. Med. 214, 113-120. https://doi.org/10.1620/tjem.214.113 (2008). 
23. Zhirnov, O. P. \& Ovcharenko, A. V. Pharmaceutical aerosol composition and application thereof for treatment and prophylaxis of viral diseases. US5723439A (1998).

24. Smee, D. F., Wong, M. H., Bailey, K. W. \& Sidwell, R. W. Activities of oseltamivir and ribavirin used alone and in combination against infections in mice with recent isolates of influenza A (H1N1) and B viruses. Antivir. Chem. Chemother. 17, 185-192. https:// doi.org/10.1177/095632020601700403 (2006).

25. Fritz, H. \& Jochum, M. Aprotinin and its target enzymes in vitro and in vito. in 9th Annual Meeting of the International Society for Heart Transplantation (eds D.E. Birnbaum \& H.E. Hoffmeister) 42-51 (Schattauer, 1990).

26. Davis, R. \& Whittington, R. Aprotinin. NDrugs 49, 954-983. https://doi.org/10.2165/00003495-199549060-00008 (1995).

27. Zhirnov, O. P., Ikizler, M. R. \& Wright, P. F. Cleavage of influenza A virus hemagglutinin in human respiratory epithelium is cell associated and sensitive to exogenous antiproteases. J. Virol. 76, 8682. https://doi.org/10.1128/JVI.76.17.8682-8689.2002 (2002).

28. Zhirnov, O. P., Matrosovich, T. Y., Matrosovich, M. N. \& Klenk, H.-D. Aprotinin, a protease inhibitor, suppresses proteolytic activation of pandemic H1N1v influenza virus. Antivir. Chem. Chemother. 21, 169-174. https://doi.org/10.3851/IMP1715 (2011).

29. Ovcharenko, A. V. \& Zhirnov, O. P. Aprotinin aerosol treatment of influenza and paramyxovirus bronchopneumonia of mice. Antiviral Res. 23, 107-118. https://doi.org/10.1016/0166-3542(94)90038-8 (1994).

30. Bertram, S., Glowacka, I., Steffen, I., Kühl, A. \& Pöhlmann, S. Novel insights into proteolytic cleavage of influenza virus hemagglutinin. Rev. Med. Virol. 20, 298-310. https://doi.org/10.1002/rmv.657 (2010).

31. Böttcher, E. et al. Proteolytic activation of influenza viruses by serine proteases TMPRSS2 and HAT from human airway epithelium. J. Virol. 80, 9896. https://doi.org/10.1128/JVI.01118-06 (2006).

32. Zhirnov, O. P., Ovcharenko, A. V. \& Bukrinskaya, A. G. Myxovirus replication in chicken embryos can be suppressed by aprotinin due to the blockage of viral glycoprotein cleavage. J. Gen. Virol. 66, 1633-1638. https://doi.org/10.1099/0022-1317-66-7-1633 (1985).

33. Bojkova, D. et al. Aprotinin inhibits SARS-CoV-2 replication. Cells 9, 2377. https://doi.org/10.3390/cells9112377 (2020).

34. Peacock, P. T., James, J., Sealy, E. J. \& Iqbal, M. A global perspective on H9N2 avian influenza virus. Viruses 11, 620. https://doi. org/10.3390/v11070620 (2019).

35. Nam, J.-H. et al. Emergence of mammalian species-infectious and -pathogenic avian influenza H6N5 virus with no evidence of adaptation. J. Virol. 85, 13271. https://doi.org/10.1128/JVI.05038-11 (2011).

36. Nam, J.-H. et al. Rapid virulence shift of an H5N2 avian influenza virus during a single passage in mice. Arch. Virol. 162, 3017-3024. https://doi.org/10.1007/s00705-017-3451-9 (2017).

37. Burnham, A. J., Baranovich, T. \& Govorkova, E. A. Neuraminidase inhibitors for influenza B virus infection: Efficacy and resistance. Antiviral Res. 100, 520-534. https://doi.org/10.1016/j.antiviral.2013.08.023 (2013).

38. Emerson, T. E. Jr. Pharmacology of aprotinin and efficacy during cardiopulmonary bypass. Cardiovasc. Drug Rev. 7, 127-140. https://doi.org/10.1111/j.1527-3466.1989.tb00521.x (1989).

39. Zhirnov, O. P., Ovcharenko, A. V. \& Bukrinskaya, A. G. Suppression of influenza virus replication in infected mice by protease inhibitors. J. Gen. Virol. 65, 191-196. https://doi.org/10.1099/0022-1317-65-1-191 (1984).

40. Erasmus Medical Centre (NL) \& Istituto Zooprofilattico Sperimentale delle Venezie (IT). Mechanisms and risk factors for mutation from low to highly pathogenic avian influenza virus. EFSA Support. Publ. 14, EN-1287. https://doi.org/10.2903/sp.efsa.2017. EN-1287 (2017).

41. Mangano, D. T., Tudor, I. C. \& Dietzel, C. The risk associated with aprotinin in cardiac surgery. New Engl. J. Med. 354, 353-365. https://doi.org/10.1056/NEJMoa051379 (2006).

42. Benedetto, U. et al. Safety of perioperative aprotinin administration during isolated coronary artery bypass graft surgery: Insights from the ART (Arterial Revascularization Trial). J. Am. Heart Assoc. 7, e007570. https://doi.org/10.1161/JAHA.117.007570 (2018).

43. Olenchock, S. A. Jr. et al. Impact of aprotinin on adverse clinical outcomes and mortality up to 12 years in a registry of 3,337 patients. Ann. Thorac. Surg. 86, 560-567. https://doi.org/10.1016/j.athoracsur.2008.04.048 (2008).

44. Dietrich, W., Ebell, A., Busley, R. \& Boulesteix, A.-L. Aprotinin and anaphylaxis: analysis of 12,403 exposures to aprotinin in cardiac surgery. Ann. Thorac. Surg. 84, 1144-1150. https://doi.org/10.1016/j.athoracsur.2007.05.015 (2007).

45. Royston, D., De Hert, S., van der Linden, J., Ouattara, A. \& Zacharowski, K. A special article following the relicence of aprotinin injection in Europe. Anaesth. Crit. Care Pain Med. 36, 97-102. https://doi.org/10.1016/j.accpm.2017.02.001 (2017).

46. Furnary Anthony, P., Wu, Y., Hiratzka Loren, F., Grunkemeier Gary, L. \& Page, U. S. 3rd. Aprotinin does not increase the risk of renal failure in cardiac surgery patients. Circulation 116, I-127-I-133. https://doi.org/10.1161/CIRCULATIONAHA.106.681395 (2007).

47. Howell, N., Senanayake, E., Freemantle, N. \& Pagano, D. Putting the record straight on aprotinin as safe and effective: Results from a mixed treatment meta-analysis of trials of aprotinin. J. Thorac. Cardiovasc. Surg. 145, 234-240. https://doi.org/10.1016/j.jtcvs. 2012.07.018 (2013).

48. European Society of Anaesthesiology task force reports on place of aprotinin in clinical anaesthesia. Aprotinin: Is it time to reconsider?. Eur. J. Anaesthesiol. 32, 591-595. https://doi.org/10.1097/EJA.0000000000000284 (2015).

49. Reed, L. J. \& Muench, H. A simple method of estimating fifty per cent endpoints. Am. J. Epidemiol. 27, 493-497. https://doi.org/ 10.1093/oxfordjournals.aje.a118408 (1938).

\section{Acknowledgements}

This work was supported by Korea University Research Grants (K1605091, K1619611, K1704981, K1721091, K1800101, K1821221, K2005541, and K2023291) and Korea University Sejong Future Research Grant (K1826621).

\section{Author contributions}

J.-K.K., C.-K.L., K.L, and S.-K.P. designed the experiments and prepared the materials. J.-K.K., E.-J.S., S.-M.S., E.E., J.-H.N., and J.K. performed the experiments and analyzed the data. E.-J.S., E.E., J.-K.K. wrote the manuscript.

\section{Competing interests}

The authors declare no competing interests.

\section{Additional information}

Correspondence and requests for materials should be addressed to C.-K.L. or J.-K.K.

Reprints and permissions information is available at www.nature.com/reprints.

Publisher's note Springer Nature remains neutral with regard to jurisdictional claims in published maps and institutional affiliations. 
(c) (i) Open Access This article is licensed under a Creative Commons Attribution 4.0 International cc) License, which permits use, sharing, adaptation, distribution and reproduction in any medium or format, as long as you give appropriate credit to the original author(s) and the source, provide a link to the Creative Commons licence, and indicate if changes were made. The images or other third party material in this article are included in the article's Creative Commons licence, unless indicated otherwise in a credit line to the material. If material is not included in the article's Creative Commons licence and your intended use is not permitted by statutory regulation or exceeds the permitted use, you will need to obtain permission directly from the copyright holder. To view a copy of this licence, visit http://creativecommons.org/licenses/by/4.0/.

(C) The Author(s) 2021 